\title{
Functional characterization of the immunomodulatory properties of human urine-derived stem cells
}

\author{
Rongpei $\mathrm{Wu}^{1,2} \wedge$, Melisa Soland ${ }^{1} \wedge$, Guihua Liu ${ }^{1,3}$, Yingai Shi ${ }^{1,4} \wedge$, Chi Zhang ${ }^{2,5}$, Yiming Tang ${ }^{2}$, \\ Graça Almeida-Porada ${ }^{1} \wedge$, Yuanyuan Zhang $^{1 \wedge}$
}

${ }^{1}$ Wake Forest Institute for Regenerative Medicine, Wake Forest University, Winston Salem, NC, USA; ${ }^{2}$ Department of Urology, The First Affiliated Hospital, Sun Yat-sen University, Guangzhou, China; ${ }^{3}$ Reproductive Medical Center, Sixth Affiliated Hospital, Sun Yat-sen University, Guangzhou, China; ${ }^{4}$ The Key Laboratory of Pathobiology, Ministry of Education, College of Basic Medical Sciences, Jilin University, Changchun, China; ${ }^{5}$ Department of Urology, The Third Affiliated Hospital, Sun Yat-sen University, Guangzhou, China

Contributions: (I) Conception and design: Y Zhang, G Almeida-Porada; (II) Administrative support: Y Zhang; (III) Provision of study materials or patients: R Wu, M Soland, G Liu, Y Shi; (IV) Collection and assembly of data: R Wu, G Liu, C Zhang, Y Tang; (V) Data analysis and interpretation: R Wu, M Soland, G Liu; (VI) Manuscript writing: All authors; (VII) Final approval of manuscript: All authors.

Correspondence to: Yuanyuan Zhang. Wake Forest Institute for Regenerative Medicine, Wake Forest School of Medicine, Winston Salem, NC 27157, USA. Email: yzhang@wakehealth.edu.

Background: Urine-derived stem cells (USCs) have been widely researched as a novel cell source for stem cell therapy, but their immunomodulatory characteristics remain to be investigated. This study aimed to characterize the immunomodulatory properties of human USCs.

Methods: Human USCs were isolated from fresh voiding urine samples from healthy male donors and expanded. Their cell surface markers were characterized by flow cytometry analysis and the telomerase activities for several USCs clones were determined. The immunosuppressive potential of USCs was evaluated by the performing the mixed lymphocyte reaction (MLR) [co-culture with peripheral blood mononuclear cells (PBMNCs)] and natural killer cells (NK) cytotoxicity assay. USCs cytokines release profile was determined by using human cytokine proteome array.

Results: USCs exhibited high cell surface expression of embryonic/mesenchymal stem cells (MSCs) markers CD29, CD44, CD54, CD73, CD90, CD146, and CD166, while lacked expression of hematopoietic stem cell markers CD11, CD14, CD19, CD31, CD34, CD45, B cell marker CD79, and co-stimulatory factors CD80 and CD86, thus, exhibiting the phenotype of MSCs. MLR indicated that USCs significantly inhibited the proliferation of PBMNCs, as compared to that of the human smooth muscle cells (SMCs). In cell cytotoxicity assays, NK cells displayed less cytotoxicity against USCs than against bone marrow mesenchymal stem cells (BMSCs) and SMCs. Furthermore, upon PBMNCs stimulation, USCs secreted higher levels of immunomodulatory cytokines, including IL-6, IL-8, MCP-1, RANTES, GRO $\alpha$, and GMCSF, compared to those of BMSCs, especially when directly contact mix-culture with PBMNCs.

Conclusions: USCs secreted immunoregulatory cytokines and possessed immunomodulatory properties, comparable to those of BMSCs.

Keywords: Urine-derived stem cells (USCs); immunomodulation; cytokines; mixed lymphocyte reaction (MLR)

Submitted Jun 05, 2021. Accepted for publication Aug 02, 2021.

doi: $10.21037 /$ tau-21-506

View this article at: https://dx.doi.org/10.21037/tau-21-506

\footnotetext{
^ ORCID: Rongpei Wu, 0000-0002-8688-4294; Melisa Soland, 0000-0001-8217-9734; Yingai Shi, 0000-0002-8469-1552; Chi Zhang, 0000-00030631-808X; Yiming Tang, 0000-0003-0348-8788; Graça Almeida-Porada, 0000-0002-6715-865X; Yuanyuan Zhang, 0000-0002-5708-9718.
} 


\section{Introduction}

Immune-mediated diseases are one of the largest types of diseases clinically studied. Autoimmune diseases are pathological states in which the immune system exerts abnormal cellular or antibody responses against patient's own tissues and organs, leading to chronic inflammation (1). These diseases affect more than $5 \%$ of the worldwide population, seriously impacting on the patient's quality of life and health care costs (2). Autoimmune diseases induce both local and systemic symptoms, leading to severe outcomes, such as systemic lupus erythematosus, rheumatoid arthritis, multiple sclerosis, and type I diabetes (2). Currently autoimmune diseases are generally treated with immune suppressive agents, such as steroids and cyclosporine. However, prolonged use of these medicines has been shown to increase treatment-related morbidity and mortality (3). Thus, development of new therapeutic strategies for autoimmune diseases is urgently required.

Stem cell therapy has been demonstrated to possess the capacity to modulate the immune system and reduce the severity of the disease in animal models of autoimmune disorders (4). For instance, rheumatoid arthritis (5), multiple sclerosis (6), lupus (7), inflammatory bowel diseases (8), among other autoimmune diseases, have responded to stem cell therapy both in preclinical and clinical studies. Among all types of stem cells, mesenchymal stem cells (MSCs) are commonly used for cell therapy due to their profound immunomodulatory properties and differentiation potentials (9), to most importantly regenerate and repair tissue. MSCs are capable of differentiating into cells of the mesodermal, endodermal, and even ectodermal lineages. Furthermore, increased evidence has shown that under appropriate culture conditions, MSCs are capable of secreting growth factors and immunoprotective cytokines, used in the field of cell and organ transplantation. Importantly, MSCs are safe and do not form teratoma, compared to embryonic or induced pluripotent stem cells.

In addition, MSCs are hypoimmunogenic and can escape the recognition by all-reactive $\mathrm{T}$ cells and natural killer (NK) cells and their subsequent cytotoxicities (9). These cells are capable of inhibiting the proliferation and activity of immune effector cells, thereby ameliorating the severity of the immune disease (9). Since first identified in the bone marrow (10), MSCs have been isolated from various tissues, called tissue-derived stem cells. The most commonly used types of MSCs in preclinical and clinical studies are cells originated from the bone marrow (BMSCs) (11), umbilical cord (UC-MSCs) (12), and adipose tissue (ADSCs) (13). Although both BMSCs and ADSCs have been investigated for a long period and applied in various experimental studies and preclinical trials (14), harvesting ADSCs and BMSCs requires invasive medical procedures, which could cause potential complications and even risk to the donor's lives. The ideal MSCs cell source should be less invasive and harmful to the donor, as well as ease to harvest and expand in large quantities. Considering this requirement, in 2008, Zhang et al. first demonstrated that stem cells can be isolated from fresh urine sample (15), termed as urine-derived stem cells (USCs). These cells have similar characteristics to MSCs $(11,16)$, can be efficiently induced into ectodermal, mesodermal, and endodermal lineages. Despite a few cells have a "rice-grain-like" morphology in the urine, they can consecutively proliferate up to 20 passages, with a doubling rate of more than 60 (16). These cells most likely originate from the parietal cell interface of the renal glomerulus and express the corresponding markers (16). More than half of USCs clones possess high telomerase activity and proliferation ability; large quantities can be obtained from a single cell $(3,17)$. Importantly, USCs can be isolated from fresh voiding urine and propagated through a simple, noninvasive, and low-cost manipulation, making them more attractive for tissue regeneration.

Therefore, USCs can be a novel alternative cell source for stem cell therapy (18). However, the immunomodulatory properties of USCs have not been fully investigated thus far. Hence, the purpose of this study was to characterize the immunomodulatory properties of human USCs.

We present the following article in accordance with the MDAR reporting checklist (available at https://dx.doi. org/10.21037/tau-21-506).

\section{Methods}

\section{Ethics statements}

This study was approved by the Wake Forest University institutional review board (IRB00014033). Written informed consents have been obtained and were approved by Wake Forest University institutional review board. The study was conducted in accordance with the Declaration of Helsinki (as revised in 2013).

\section{Isolation of buman USCs}

Fresh voiding urine samples were collected from three 
healthy male donors of 28-, 35-, and 41-year-old, respectively. For isolation of USCs, the urine samples were centrifuged at $500 \times \mathrm{g}$ for $5 \mathrm{~min}$ at room temperature. The cell pellets were re-suspended and then seeded into 24-well tissue culture plates (Becton Dickinson, Franklin Lakes, NJ, USA). The culture medium contained Keratinocyte Serum Free Medium (KSFM, Gibco, Gaithersburg, MD, USA) and Dulbecco's Modified Eagle Medium (DMEM, high glucose, Gibco, Gaithersburg, MD, USA) at a 1:1 ratio with $5 \%$ fetal bovine serum (FBS, Gibco, Gaithersburg, MD, USA). Urine-derived cells were isolated and characterized as previously described (16). USCs at passages 2-5 were used for following studies.

\section{Cell culture}

Human peripheral blood mononuclear cells (PBMNCs, normal human, ATCC PCS-800-011) from two different donors were purchased from ATCC (American Type Culture Collection, MD, USA). PBMNCs were thaw in the Hank's Balanced Salt Solution medium (HBSS, ATCC 302213, MD, USA) and directly used in the experiments. Human BMSCs and human SMCs were purchased from Lonza (BMSCs: PT-2501, SMCs: CC-2533, Lonza, Swiss).

\section{Flow cytometry for stem cell surface markers}

USCs at passage 2 were stained with specific antibodies against human CD11b-FITC, CD14-APC, CD19-FITC, CD29-PE, CD31-FITC, CD34-FITC, CD40-FITC, CD44-FITC, CD45-FITC, CD54-PE, CD73-PE, CD79PE, CD90-FITC, CD133-PE, CD140b-PE, CD146-PE, CD166-PE, CD80-FITC, CD86-PE, HLA-ABC-FITC, HLA-G-FITC, and HLA-DR-FITC. Briefly, cells were trypsinized and a total of $5.0 \times 10^{5}$ cells were re-suspended in ice-cold PBS containing 1\% bovine serum albumin (BSA, 23210, Thermo Fisher scientific, Rockford, IL, USA). Fluorochrome-conjugated antibodies (Table 1) were added to $50 \mu \mathrm{L}$ of the cell suspension containing $1 \times \mathrm{PBS}$ and $1 \% \mathrm{BSA}$, the mix was incubated on ice for $30 \mathrm{~min}$ in the dark. IgG1-PE, IgG1-FITC, IgG2b-FITC, and IgG1APC conjugated isotype control antibodies were used to determine the background fluorescence. Cells were then washed twice in wash buffer, passed through a $70 \mu \mathrm{m}$ filter, and detected by flow cytometry (FACS Calibur BD Biosciences, Franklin Lakes, NJ, USA), procedures were repeated three times. The data were analyzed by using FlowJo vX software (Tree Star, Ashland, OR, USA).

\section{Telomerase activity assay}

The telomerase activity of USCs was directly associated with their proliferative potential according to our prior research (16). Otherwise, does immune characters associate with telomerase activity not clear yet. The telomerase activities of the USCs clones were determined by the telomeric repeat amplification protocol assay, using the Telo TAGGG Telomerase PCR ELISA ${ }^{\text {plus }}$ kit (Roche, Germany) and according to manufacturer's protocol. To ensure that each USCs clone was originated from a single cell, only wells in multi-well plates with a single spindle shape cell were selected for expansion. The telomerase activity of human BMSCs and SMCs, as well as of HEK293 cell line (ATCC CRL-1573, MD, USA) as control, were also measured. A total of $2 \times 10^{5}$ cells at passage 2 were collected for DNA extraction.

After DNA amplification by PCR, the products were subjected to hybridization and ELISA procedures. The results were reported by subtracting the absorbance of the samples at $450 \mathrm{~nm}$. The level of telomerase activity in a given sample was determined by comparing the signal originated by the sample to the signal obtained using a known amount of a control template in the kit. Relative telomerase activities (RTA) of samples were obtained using the following formula:

$$
\mathrm{RTA}=[(\mathrm{AS}-\mathrm{AHt}) / \mathrm{AIs}] /[(\mathrm{AC}-\mathrm{ALb}) / \mathrm{AIsC}] \times 100
$$

RTA: relative telomerase activity; AS: absorbance of sample; AHt: absorbance of heat-treated sample; AIs: absorbance of internal standard of the sample; AC: absorbance of control template; ALb: absorbance of lysis buffer; AIsC: absorbance of internal standard of the control template. Internal standard and control template were all provided in the kit.

\section{Mixed lymphocyte reaction (MLR)}

$\mathrm{USC}^{-\mathrm{TA}^{+}}$(USCs clone with high telomerase activity), USC$\mathrm{TA}^{-}$(USCs clone with low telomerase activity), BMSCs, and SMCs were used as stimulator cells; meanwhile, human PBMNCs from two healthy donors with different blood types were used as responder cells. Approximately $1 \times 10^{4}$ 
Table 1 Antibodies used in this study

\begin{tabular}{|c|c|c|c|c|}
\hline Antibody & Type & Dilution & Source (company) & Catalog No. \\
\hline CD14-APC & Mouse mAb & $1: 10$ & BD Pharmingen ${ }^{\mathrm{TM}}$ & 561708 \\
\hline CD19-FITC & Mouse mAb & $1: 10$ & BD Pharmingen ${ }^{\mathrm{TM}}$ & 560994 \\
\hline CD29-PE & Mouse mAb & $1: 10$ & BD Pharmingen ${ }^{\mathrm{TM}}$ & 555443 \\
\hline CD34-FITC & Mouse mAb & $1: 10$ & BD Pharmingen ${ }^{\mathrm{TM}}$ & 560942 \\
\hline CD40-FITC & Mouse mAb & $1: 10$ & BD Pharmingen ${ }^{\mathrm{TM}}$ & 556624 \\
\hline CD44-FITC & Mouse mAb & $1: 10$ & BD Pharmingen ${ }^{\mathrm{TM}}$ & 555478 \\
\hline CD45-FITC & Mouse mAb & $1: 10$ & BD Pharmingen ${ }^{\mathrm{TM}}$ & 560976 \\
\hline CD79-PE & Mouse mAb & $1: 10$ & BD Pharmingen ${ }^{\mathrm{TM}}$ & 563777 \\
\hline CD90-FITC & Mouse mAb & $1: 10$ & BD Pharmingen ${ }^{\mathrm{TM}}$ & 555595 \\
\hline CD140b-PE & Mouse mAb & $1: 10$ & BD Pharmingen ${ }^{\mathrm{TM}}$ & 558821 \\
\hline CD146-PE & Mouse mAb & $1: 10$ & BD Pharmingen ${ }^{\mathrm{TM}}$ & 550315 \\
\hline CD166-PE & Mouse mAb & $1: 10$ & BD Pharmingen ${ }^{\mathrm{TM}}$ & 560903 \\
\hline CD80-FITC & Mouse mAb & $1: 10$ & $\mathrm{BD}$ Pharmingen ${ }^{\mathrm{TM}}$ & 557226 \\
\hline CD86-PE & Mouse mAb & $1: 10$ & $\mathrm{BD}$ Pharmingen ${ }^{\mathrm{TM}}$ & 557344 \\
\hline HLA-ABC-FITC & Mouse mAb & $1: 10$ & $\mathrm{BD}$ Pharmingen ${ }^{\mathrm{TM}}$ & 555552 \\
\hline
\end{tabular}

stimulator cells of each type were seeded in triplicate into a 96-well flat-bottom plate (BD Falcon, USA) with Mesenchymal Stem Cell Growth Medium (MSCGM, Thermo Fisher Scientific-Gibco, Carlsbad, CA, USA). The cells were cultured to near confluency, and then treated with $5 \mu \mathrm{g} / \mathrm{mL}$ mitomycin C (Roche Applied Science, Mannheim, Germany) at $37{ }^{\circ} \mathrm{C}$ for $2.5 \mathrm{~h}$ in a humidified incubator with $5 \% \mathrm{CO}_{2}$ to prevent further proliferation. After incubation, cells were washed three times with DMEM. For co-culture experiments, $1 \times 10^{5}$ responder cells were added to each well, in a final volume of $100 \mu \mathrm{L}$ of DMEM with $10 \%$ FBS. Cultures were incubated at $37^{\circ} \mathrm{C}$ for 5 days in $5 \% \mathrm{CO}_{2}$ and $100 \%$ humidity and checked with inverted microscope before further experiment. On the fifth day, $10 \mu \mathrm{L}$ of Bromodeoxyuridine (BrdU, Roche Applied Science, Mannheim, Germany) was added to each well to a final concentration of $10 \mu \mathrm{M}$. After incubation for additional $24 \mathrm{~h}$ at $37{ }^{\circ} \mathrm{C}$ in $5 \% \mathrm{CO}_{2}$ and $100 \%$ humidity, DNA synthesis was assessed with the BrdU cell proliferation colorimetric ELISA Kit (Roche Applied Science, Mannheim, Germany), according to the manufacturer's instructions. Newly synthesized BrdU-DNA was quantified using a scanning multi-well spectrophotometer (Bio-Rad, Hercules, CA, USA). One-way MLR with single donor PBMNCs as responder cells, and two-way MLR with aliquot half dose of two different donors' PBMNCs as responder cells were detected in the same multi-well plate, respectively. All the reactions were set up in triplicate per experiment and the experiment was repeated 3 times.

\section{NK cytotoxicity assay}

Human NK-92 MI cells were purchased from ATCC (ATCC CRL-2408, MD, USA). The NK cytotoxicity assay 
was performed using the Cyto Tox $96^{\circledR}$ Non-Radioactive Cytotoxicity Assay kit (Promega, WI, USA), according to the manufacturer's protocol. $50 \mu \mathrm{L}$ USC-TA ${ }^{+}$, USC$\mathrm{TA}^{-}, \mathrm{BMSCs}$, and SMCs in $\alpha$-MEM complete medium without phenol red (Gibco, Gaithersburg, MD, USA) at the concentration of $1 \times 10^{5}$ cells $/ \mathrm{mL}$ were added into a roundbottom 96-well tissue culture microplate (BD Falcon, USA) as target cells, NK-92 MI cells (effectors) at E:T ratios of 20:1, 10:1, 5:1, and 1:1 were added in the wells of target cells with the final volume of $100 \mu \mathrm{L}$. The plate was centrifuged at $250 \times \mathrm{g}$ for $4 \mathrm{~min}$, followed by incubation at $37{ }^{\circ} \mathrm{C}, 5 \% \mathrm{CO}_{2}$ for $4 \mathrm{~h}$. The plate was then centrifuged at $250 \times \mathrm{g}$ for $4 \mathrm{~min}, 50 \mu \mathrm{L}$ of each supernatant was transferred to another flat-bottom 96-well microplate containing $50 \mu \mathrm{L}$ of substrate mix (CytoTox $96{ }^{\circledR}$ Reagent), and the microplate was incubated for $30 \mathrm{~min}$ at room temperature in the dark. Absorbance at $490 \mathrm{~nm}$ was determined using scanning multi-well spectrophotometer (Bio-Rad, Hercules, CA, USA) immediately after adding $50 \mu \mathrm{L}$ of Stop Solution. The percentage of cell lysis was calculated based on the following equation:

$$
\text { Target cell lysis } \%=\frac{\text { test release- } \text { spontaneous release }}{\text { maximum release- } \text { spontaneous release }} \times 100 \%
$$

Spontaneous release corresponded to the cytotoxicity of target cells alone in the medium, while target cell maximum release was obtained by adding $10 \mu \mathrm{L}$ of $9 \%$ Triton $\mathrm{X}-100$ solution to the cells and incubating the mix for $45 \mathrm{~min}$ at $37^{\circ} \mathrm{C}$, prior to harvesting the supernatants. All the reactions were performed in triplicate per experiment and each experiment was repeated 3 times.

\section{Cytokine proteome array}

USC-TA ${ }^{+}$, USC-TA ${ }^{-}$, BMSCs were cultured alone, cultured in direct contacting with PBMNCs, and with PBMNCs in $0.4 \mu \mathrm{m}$ Millicell hanging cell culture transwell insert (Millipore, USA), respectively, to determine the cytokines and chemokines released by the cells. The levels of cytokines and chemokines were tested in cell culture supernatants using the Human Cytokine Array Panel A kit (R\&D Systems Inc., MN, USA), according to the manufacturer's instructions. The array panel template is showed in Table 2. Aliquot of $1 \mathrm{~mL}$ from each supernatant was incubated with $15 \mu \mathrm{L}$ of human cytokine antibody cocktail for $1 \mathrm{~h}$ at room temperature, and the mixture was then added to previously blocked nitrocellulose membranes and incubated at $4{ }^{\circ} \mathrm{C}$ overnight on a rocking platform shaker. On the next day, the membranes were washed three times (10 min each) with $1 \times$ wash buffer and incubated with Streptavidin-HRP and Chemi Reagent Mix for $30 \mathrm{~min}$ at room temperature on a rocking platform shaker. The immunoblot images were visualized and captured using the LAS-3000 Image Analyzer (Fujifilm, Japan). Quantity One software (Bio-Rad, CA, USA, RRID:SCR_014280) was used to quantify the cytokine levels.

\section{Statistical analysis}

Data are expressed as the mean \pm standard deviation (SD). Means among groups were compared by one-way ANOVA or Kruskal-Walis test (if appropriate) and the corresponding post-hoc test (Fisher's LSD test for ANOVA). The significance level was set at $\mathrm{P}$ value $<0.05$ for all tests. Statistical analyses were performed using the SPSS software version 25 (IBM Corporation, NY, USA).

\section{Results}

\section{USCs expressed embryonic/MSCs markers}

We determined the cell markers present on the surface of isolated USCs by flow cytometry analysis (Table 3). According to our results, the USCs major population expressed typical embryonic/MSCs markers, such as CD29 (100\%), CD44 (100\%), CD54 (98.9\%), CD73 (99.6\%), CD90 (75.5\%), CD146 (99.2\%), and CD166 (99.4\%); meanwhile, a small population of USCs expressed CD140 (10.7\%). In contrast, USCs did not present hematopoietic stem cell markers CD11b (0.98\%), CD14 (1.55\%), CD19 (0.914\%), CD31 (0.861\%), CD34 (1.02\%), CD45 (0.737\%), and B cell marker CD79 (1.05\%) on their surfaces.

In addition, USCs tested lightly positive for the costimulatory factor CD40 (28.3\%) and negative for costimulatory factors CD80 (0.86\%) and CD86 (0.63\%). Regarding major histocompatibility complex (MHC) antigens, most of USCs contained HLA-ABC markers (99.8\%) but the cell surface expression of HLA-DR $(0.642 \%)$ and HLA-G $(0.996 \%)$ antigens was negligible (Figure 1). These data suggested that the isolated USCs indeed expressed MSCs markers, indicating they may possess MSCs characteristics.

\section{USCs presented varied telomerase activity}

After determining the telomerase activity of the USCs 
Table 2 Human cytokine array panel a coordinates

\begin{tabular}{|c|c|c|}
\hline Coordinate & Target/control & Alternate nomenclature \\
\hline $\mathrm{A} 1, \mathrm{~A} 2$ & Reference Spot & - \\
\hline A3, A4 & C5/C5a & Complement Component 5/5a \\
\hline A5, A6 & CD40 ligand & CD154 \\
\hline A7, A8 & G-CSF & $\operatorname{CSF} \beta, \mathrm{CSF}-3$ \\
\hline A9, A10 & GM-CSF & $\mathrm{CSF} \alpha, \mathrm{CSF}-2$ \\
\hline $\mathrm{A} 11, \mathrm{~A} 12$ & $\mathrm{GRO} \alpha$ & CXCL1 \\
\hline A13, A14 & I-309 & CCL1 \\
\hline A15, A16 & sICAM-1 & CD54 \\
\hline A17, A18 & $\mathrm{IFN}-\gamma$ & Type II IFN \\
\hline $\mathrm{A} 19, \mathrm{~A} 20$ & Reference Spot & - \\
\hline B3, B4 & $\mathrm{IL}-1 \alpha$ & IL-1F1 \\
\hline B5, B6 & IL-1 $\beta$ & IL-1F2 \\
\hline B7, B8 & IL-1ra & IL-1F3 \\
\hline B9, B10 & IL-2 & - \\
\hline $\mathrm{B} 11, \mathrm{~B} 12$ & IL-4 & - \\
\hline B13, B14 & IL-5 & - \\
\hline $\mathrm{B} 15, \mathrm{~B} 16$ & IL-6 & - \\
\hline $\mathrm{B} 17, \mathrm{~B} 18$ & IL-8 & CXCL8 \\
\hline $\mathrm{C} 3, \mathrm{C} 4$ & IL-10 & - \\
\hline C5, C6 & IL-12 p70 & - \\
\hline $\mathrm{C} 7, \mathrm{C} 8$ & IL-13 & - \\
\hline $\mathrm{C9}, \mathrm{C} 10$ & IL-16 & LCF \\
\hline C11, C12 & IL-17 & - \\
\hline C13, C14 & IL-17E & - \\
\hline C15, C16 & IL-23 & - \\
\hline C17, C18 & IL-27 & - \\
\hline D3, D4 & IL-32 $\alpha$ & - \\
\hline D5, D6 & IP-10 & CXCL10 \\
\hline D7, D8 & I-TAC & CXCL11 \\
\hline D9, D10 & MCP-1 & CCL2 \\
\hline D11, D12 & MIF & GIF, DER6 \\
\hline D13, D14 & $\mathrm{MIP}-1 \alpha$ & CCL3 \\
\hline D15, D16 & MIP- $1 \beta$ & CCL4 \\
\hline D17, D18 & Serpin E1 & PAI-1 \\
\hline $\mathrm{E} 1, \mathrm{E} 2$ & Reference Spot & - \\
\hline
\end{tabular}

Table 2 (continued)
Table 2 (continued)

\begin{tabular}{lcc}
\hline Coordinate & Target/control & Alternate nomenclature \\
\hline E3, E4 & RANTES & CCL5 \\
E5, E6 & SDF-1 & CXCL12 \\
E7, E8 & TNF- $\alpha$ & TNFSF1A \\
E9, E10 & sTREM-1 & - \\
E19, E20 & Negative control & - \\
\hline
\end{tabular}

samples, specific clones were selected from three donors ( 2 clones/donor). Each donor provided two USCs clones, one with high telomerase activity (USC-TA ${ }^{+}$) and another one with low telomerase activity (USC-TA'), that were used for further studies.

\section{USCs inbibited the proliferation of PBMNCs in MLR}

To determine whether USCs had immunomodulatory properties, USC-TA ${ }^{+}$and USC-TA ${ }^{-}$were co-cultured with PBMNCs from two different donors (one-way MLR and two-way MLR) by analyzing their immunosuppressive potentials towards PBMNCs. As shown in Figure 2, both $\mathrm{USC}^{-\mathrm{TA}^{+}}$and USC-TA${ }^{-}$elicited a higher inhibition on the MLR reactions, when compared to SMCs and BMSCs, although the difference between USC-TA ${ }^{+}$, USC-TA and BMSCs was not significant. The difference between USC-TA ${ }^{+} / \mathrm{USC}^{-\mathrm{TA}^{-}}$and SMCs was significant when using the PBMNCs no matter from one specific donor or two different donors (all $\mathrm{P}<0.05$ ). That reveal both $\mathrm{USC}^{-\mathrm{TA}^{+}}$and USC-TA ${ }^{-}$had a higher capacity to inhibit the proliferation of PBMNCs, when compared to that of SMCs control. These results suggested that both USC-TA ${ }^{+}$ and USC-TA ${ }^{-}$possessed comparable immunosuppressive potentials to that of BMSCs.

\section{USCs inbibited NK cytotoxicities}

Next, we further evaluated the immunomodulatory properties of USCs with NK cytotoxicity assays. The results showed that both USC-TA ${ }^{+}$and USC-TA ${ }^{-}$induced less cytotoxicity than SMCs at E:T ratios of 20:1, 10:1, 5:1, and $1: 1$, although only the difference between USC-TA ${ }^{+}$ and BMSCs at the E:T ratio 10:1 was significant $(\mathrm{P}=0.013)$. These results suggested that USCs possessed a comparable or even a little better immunomodulatory property than that of BMSCs (Figure 3). 
Table 3 Flow cytometry for cell surface markers of USCs

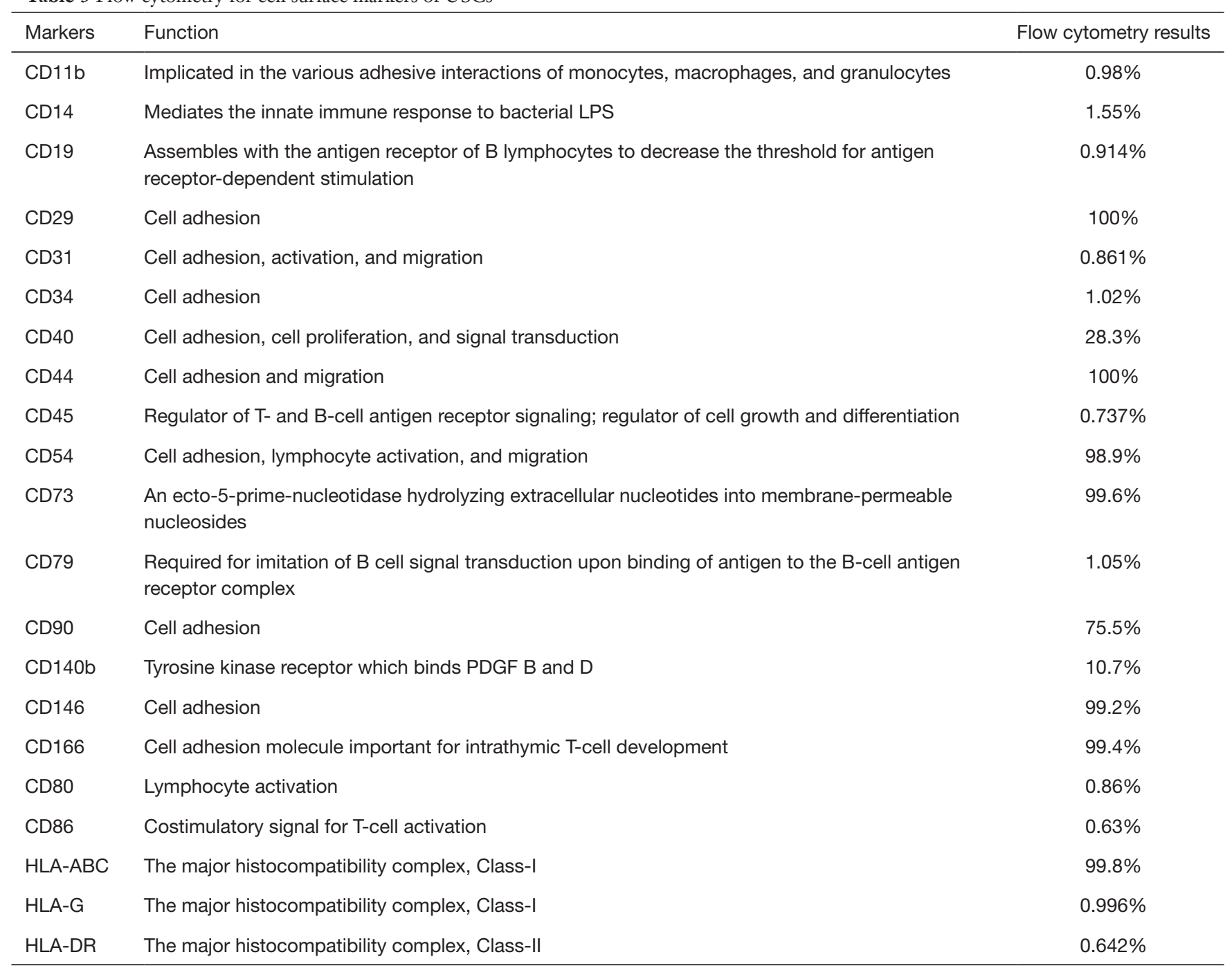

LPS, lipopolysaccharide; PDGF, platelet derived growth factor.

\section{USCs exhibited a specific cytokine release profile}

Finally, the cytokines and chemokines released by USCs to the supernatants when cultured in absence of PBMNCs and in direct or indirect contact with PBMNCs were evaluated (Figure 4A). After PBMNCs stimulation, the levels of two immunoregulatory cytokines, IL-6, IL-8, and one chemokine MCP-1 (monocyte chemotactic protein-1) were significantly up-regulated in BMSCs, USC-TA ${ }^{+}$, and $\mathrm{USC}^{-\mathrm{TA}^{-}}$(all $\mathrm{P}<0.05$ ). In addition, the direct contact mixed culture with $\mathrm{PBMNCs}$ induced a higher release of IL- 8 and MCP-1 by USC-TA ${ }^{+}$and USC-TA', when compared to that obtained from the same cells culturing supernatant of indirect mixed cultures with PBMNCs in hanging transwell insert. The expression of other cytokines, including RANTES (regulated upon activation, normal T cell expressed and presumably secreted), GRO $\alpha$ (growthregulated oncogene $\alpha$ ), and GM-CSF (granulocytemacrophage colony stimulating factor) was also upregulated in BMSCs, USC-TA ${ }^{+}$, and $\mathrm{USC}^{-\mathrm{TA}^{-} \text {upon }}$ $\mathrm{PBMNCs}$ stimulation, especially with direct contact mix culture (Figure 4B,4C).

\section{Discussion}

This study demonstrated that human USCs express immunological markers and possess immunomodulatory properties in similar levels to MSCs. USCs significantly 

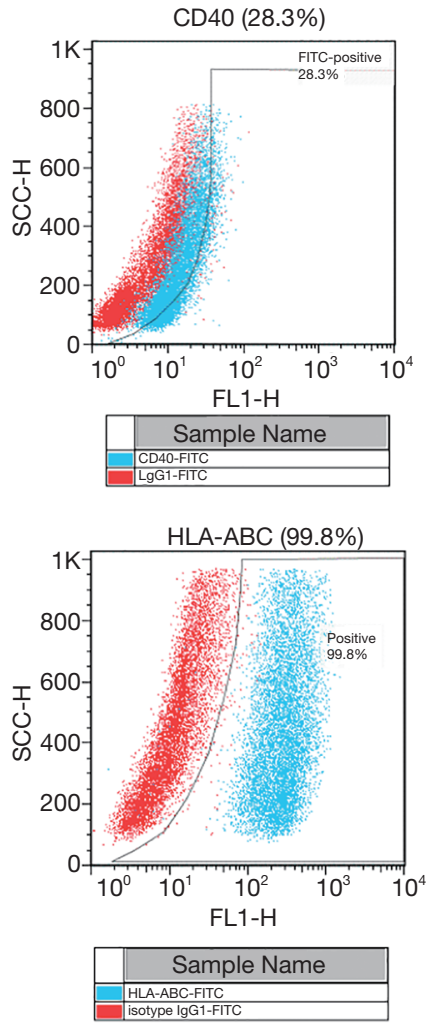
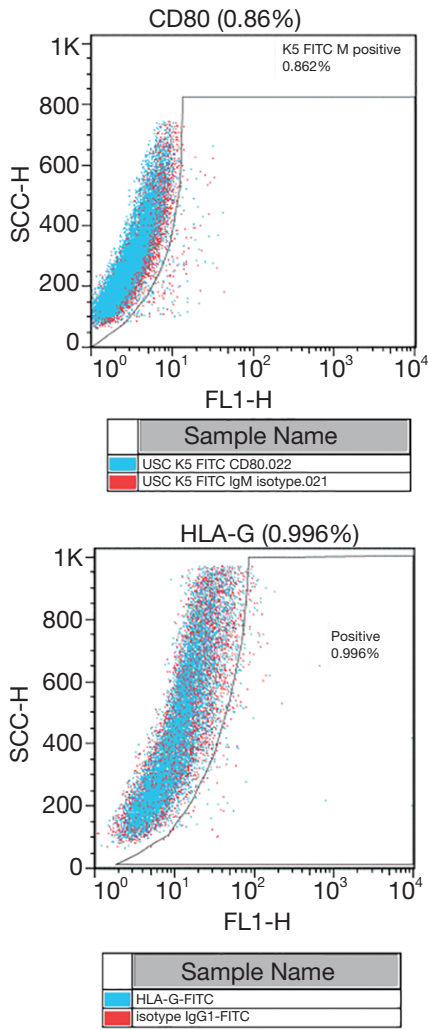
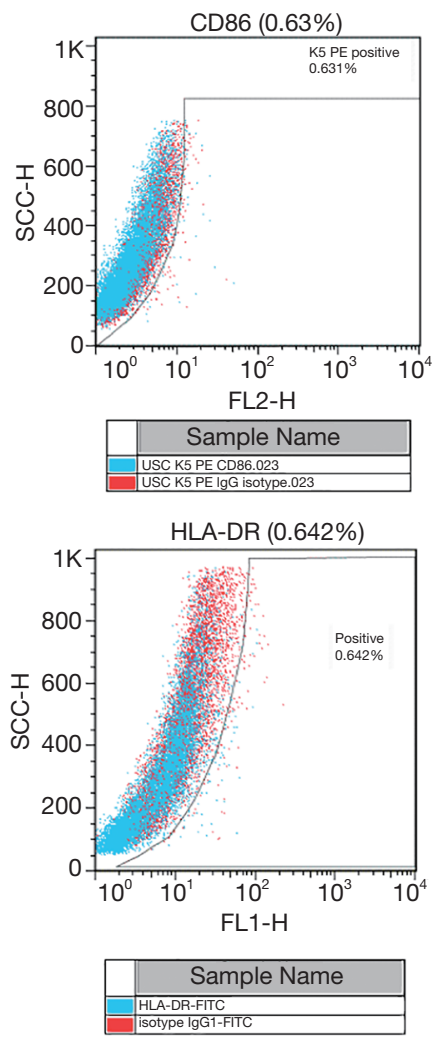

Figure 1 USCs surface markers were determined by flow cytometry. Flow data showed the detection of co-stimulatory molecules CD40, CD80, and CD86 and immunological markers HLA-ABC, HLA-G, and HLA-DR.

inhibited the proliferation of PBMNCs in a co-culture system and inhibited the cytotoxicity activity of NK cells. Furthermore, USCs significantly increased the secretion of immunoregulatory cytokines such as IL-6, IL-8 and the immunoregulatory chemokine MCP-1 when the cells were contacting with PBMNCs.

From the histological point of view, USCs constitute a renal parietal cell linage and are different from MSCs; however, USCs appear to have similar characteristics to MSCs. The flow cytometry data showed that almost the whole isolated USCs population homogenously expressed embryonic/mesenchymal stem cell markers, but not hematopoietic and B cell markers, suggesting that isolated USCs possessed the MSCs potency. This surface expression pattern agreed with the ones described in previous studies of USCs $(16,19,20)$. Otherwise, CD80 and CD86 are costimulatory molecules located on the antigen presenting cells which mediate T-cell inhibitory signals by interacting with CD152 on activated T cells (21). Our results showed both BMSCs and USCs tested negative for the presence of
CD80 and CD86. Regarding immunological markers, USCs contained HLA-ABC antigens (major histocompatibility complex class I, MHC-I), while lacked the expression of HLA-DR (MHC-II) and HLA-G (MHC-I), similarly to BMSCs (22). HLA-DR mismatch is an important factor leading to graft loss after transplantation (23). The absence of co-stimulatory molecules and MHC-II markers on cultured USCs suggested that they possessed hypoimmunogenic properties, allowing them to escape their recognition by the recipient's immune system. Taken together, these data suggested that the isolated USCs expressed immunomodulatory surface markers, highly resembling to those of MSCs. USCs shared similar stem cell markers with BMSCs, but also expressed different ones, suggesting that USCs might be a new type of adult stem cells with hypoimmunogenic characteristics that require further characterization.

MSCs, even from different origins, possess significant immunomodulatory potential. Since USCs may be an optimal source for stem cell therapy in cases of immune 

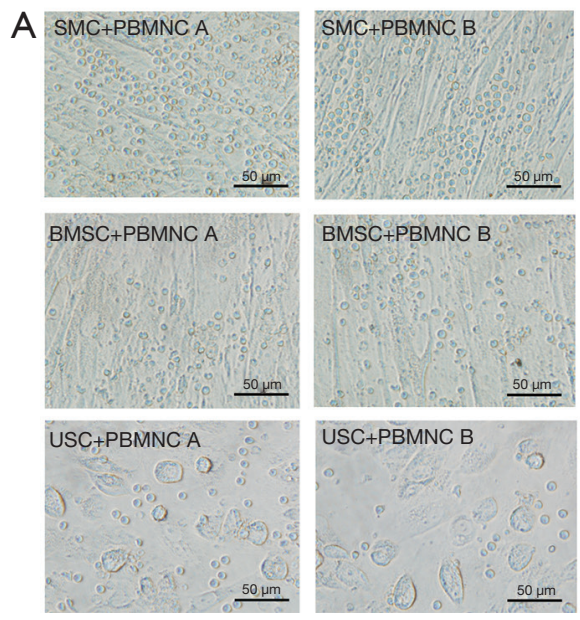
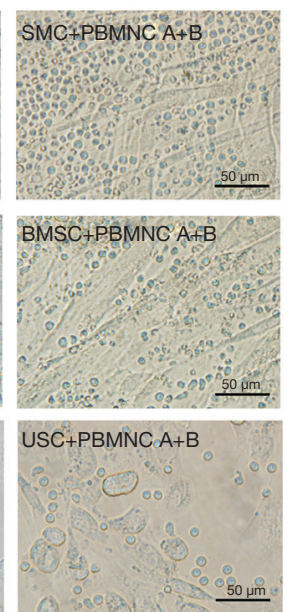

B

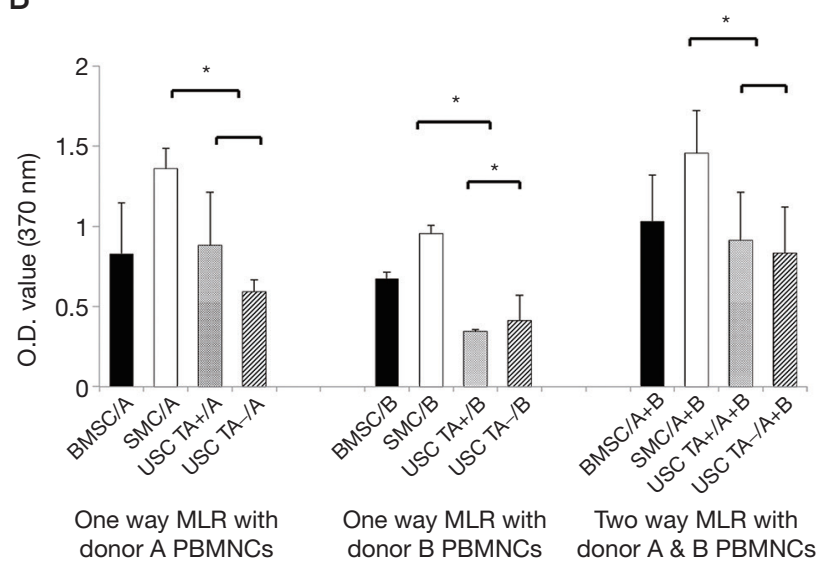

Figure 2 USCs inhibited the proliferation of PBMNCs in MLR. USC-TA+, USC-TA-, BMSCs, and SMCs were used as stimulator cells and human PBMNCs from two healthy donors were used as responder cells. One-way MLR with single donor PBMNCs (donor A or B) as responder cells and two-way MLR with aliquot half dose of two different donors' PBMNCs (donor A and B) as responder cells were detected in the same multi-well plate. Cultures were incubated at $37{ }^{\circ} \mathrm{C}$ for 5 days in $5 \% \mathrm{CO}_{2}$ and $100 \%$ humidity, the cells were checked with inverted microscope on the 5 th day before further experiment. There were different cell densities of PBMNCs be noticed in different wells for 5 days mix-culture, Scale bar $=50 \mu \mathrm{m}$ (A). The proliferation of PBMNCs was assessed with the BrdU cell proliferation colorimetric ELISA. Newly synthesized BrdU-DNA was quantified using a scanning multi-well spectrophotometer (B). *, $\mathrm{P}<0.05$, posthoc paired-comparisons. MLR, mixed lymphocyte reaction; BMSC, human bone marrow mesenchymal stem cells; SMC, human smooth muscle cells; USC TA+, human urine derived stem cells with high telomerase activity; USC TA-, human urine derived stem cells with low telomerase activity; PBMNC, human peripheral blood mononuclear cells; SMC + PBMNC A, one-way mixed lymphocyte reaction as human smooth muscle cells mixed with human peripheral blood mononuclear cells from donor A; SMC + PBMNC B, one-way mixed lymphocyte reaction as human smooth muscle cells mixed with human peripheral blood mononuclear cells from donor B; SMC + PBMNC $\mathrm{A}+\mathrm{B}$, two-way mixed lymphocyte reaction as human smooth muscle cells mixed with aliquot half dose of two different donors' human peripheral blood mononuclear cells from donor A and donor B; BMSC + PBMNC A, one-way mixed lymphocyte reaction as human bone marrow mesenchymal stem cells mixed with human peripheral blood mononuclear cells from donor A; BMSC + PBMNC B, one-way mixed lymphocyte reaction as human bone marrow mesenchymal stem cells mixed with human peripheral blood mononuclear cells from donor B; BMSC + PBMNC A + B, two-way mixed lymphocyte reaction as human bone marrow mesenchymal stem cells mixed with aliquot half dose of two different donors' human peripheral blood mononuclear cells from donor A and donor B; USC + PBMNC A, one-way mixed lymphocyte reaction as human urine derived stem cells mixed with human peripheral blood mononuclear cells from donor A; USC + PBMNC B, one-way mixed lymphocyte reaction as human urine derived stem cells mixed with human peripheral blood mononuclear cells from donor B; USC + PBMNC A + B, two-way mixed lymphocyte reaction as human urine derived stem cells mixed with aliquot half dose of two different donors' human peripheral blood mononuclear cells from donor A and donor B.

dysfunction (4), we evaluated their immunomodulatory potential. PBMNCs proliferate when co-cultured with other cells due to alloantigen stimulation (24). In this study, USCs were capable of down-regulation alloantigen-driven proliferation of PBMNCs both in one-way and two-way MLR cultures, exhibiting a comparable immunomodulatory potential to that of BMSCs. Our findings are in line with previous studies $(19,20)$. Due to the hypoimmunogenic property, MSCs can escape from the recognition by $\mathrm{T}$ cells (25), cytotoxic T lymphocytes (26), NK-T cells (27), and NK cell-mediated lysis (28). In this study, the NK cytotoxicity assay showed that both USC-TA ${ }^{+}$and USC$\mathrm{TA}^{-}$induced less NK cytotoxicity than SMCs at various E:T ratios, and the difference between USC- $\mathrm{TA}^{+}$and BMSCs at the $\mathrm{E}: \mathrm{T}$ ratio $10: 1$ was significant $(\mathrm{P}=0.013)$. Taken together, these results suggested that USCs possessed a comparable or even better immunomodulatory property than that of BMSCs.

MSCs have immunomodulatory functions which are exerted by direct cell-to-cell contacts, secretion of cytokines 


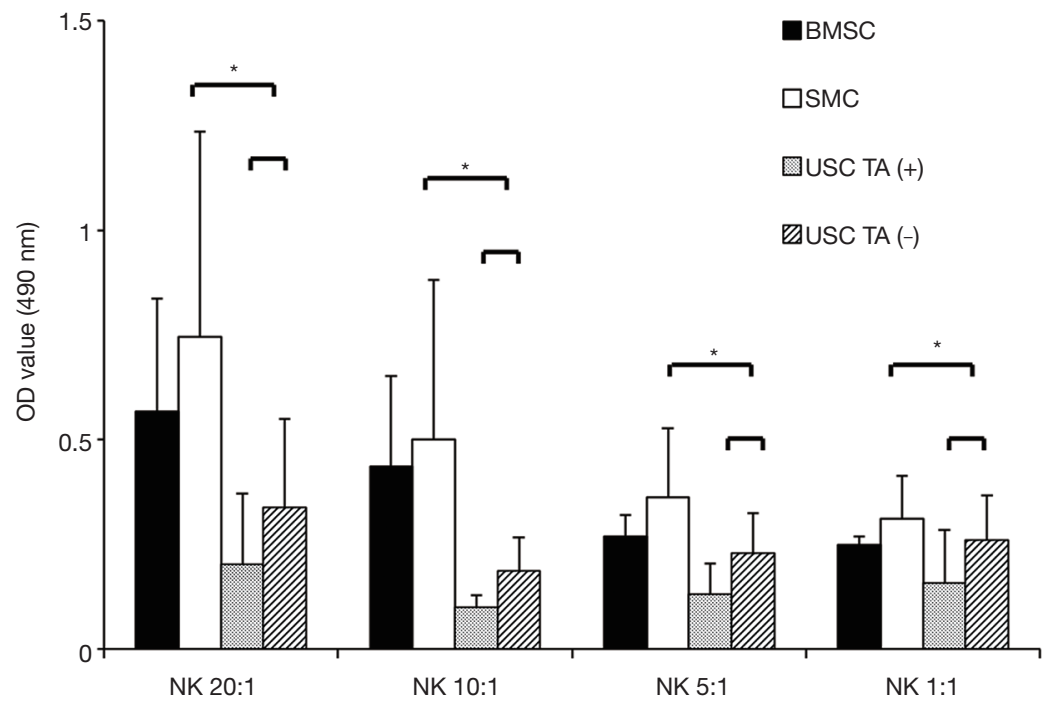

Figure 3 Co-culture with USCs inhibited the NK cytotoxicity. USC-TA+, USC-TA-, BMSCs, and SMCs (target cells, $1 \times 10^{5}$ cells/mL; $50 \mu \mathrm{L} /$ well) were co-cultured with NK-92MI cells (effectors) at E:T ratios of 20:1, 10:1, 5:1, and 1:1 for 4 h. The target cells lysis was determined by measuring the absorbance at $490 \mathrm{~nm}$, as described in Methods. * $\mathrm{P}<0.05$, post-hoc paired-comparisons. BMSC, human bone marrow mesenchymal stem cells; SMC, human smooth muscle cells; USC TA+, human urine derived stem cells with high telomerase activity; USC TA-, human urine derived stem cells with low telomerase activity; NK, human natural killer cells.

and/or by a combination of both mechanisms, effects of MSCs immunosuppressive are determined by the local conditions of the microenvironment, pro-inflammatory cytokines and chemokines may induce secretion of antiinflammatory immunosuppressive factors (29). In this study, human cytokine release arrays showed that the levels of immunoregulatory cytokines, IL-6, IL-8, and immunoregulatory chemokine $\mathrm{MCP}-1$ were significantly elevated after USC-TA ${ }^{+}$and USC- $\mathrm{TA}^{-}$stimulation with PBMNCs. In addition, the direct contacting of USCs with PBMNCs induced a higher release of IL- 8 and MCP-1 than that detected in indirect contacting culture with PBMNCs in hanging transwell insert. The elevated levels of IL-8 and MCP-1 in USCs (both $\mathrm{TA}^{+}$and $\mathrm{TA}^{-}$) were significantly higher than those in BMSCs, especially mix-culture directly contact with PBMNCs. The concentration of other cytokines, including RANTES, GRO $\alpha$, and GM-CSF were also up-regulated upon PBMNCs stimulation. These cytokines might play a role in the mechanisms underlying the USC-induced inhibition of immune cells proliferation as well as cytotoxicity effects, reveal the immunomodulatory properties of USCs, which grants further investigation.

Due to the high telomerase activity, USCs possess a highly proliferative potential that can generate a large number of cells from a single clone (17). Qin et al. previously showed that USCs with high telomerase activity can be maintained for up to 20 passages, while USCs with low telomerase activity can grow for only 8 to 10 passages (30). To address the effect of telomerase activity on the immunomodulatory properties of USCs, USC-TA ${ }^{+}$ and USC-TA ${ }^{-}$clones from the same donor were isolated and expanded. In the current study, the MLR and NK cytotoxicity assays indicated the absence of a significant difference between the inhibitory effects of USCs- $\mathrm{TA}^{+}$and USCs- $\mathrm{TA}^{-}$, suggesting that the telomerase activity seemed to have little or no effect on the immunomodulatory properties of USCs, even though the USC-TA ${ }^{+}$cells were more easily expanded to a suitable quantity for clinical usage. However, it is worth to further address in vivo whether USC- $\mathrm{TA}^{+}$can maintain their immunomodulatory properties for longer periods than USC- $\mathrm{TA}^{-}$cells.

Several limitations exist in the current study that need to be addressed in the future. Although correlation between the USCs telomerase activity and their immunomodulatory properties was not significant in this study, it is still worth to further investigate whether the telomerase activity influences the immunomodulatory properties of USCs depending on the cell passage number or donor age. In addition, even though we observed that the PBMNCs stimulation enhanced the USCs secretion of 


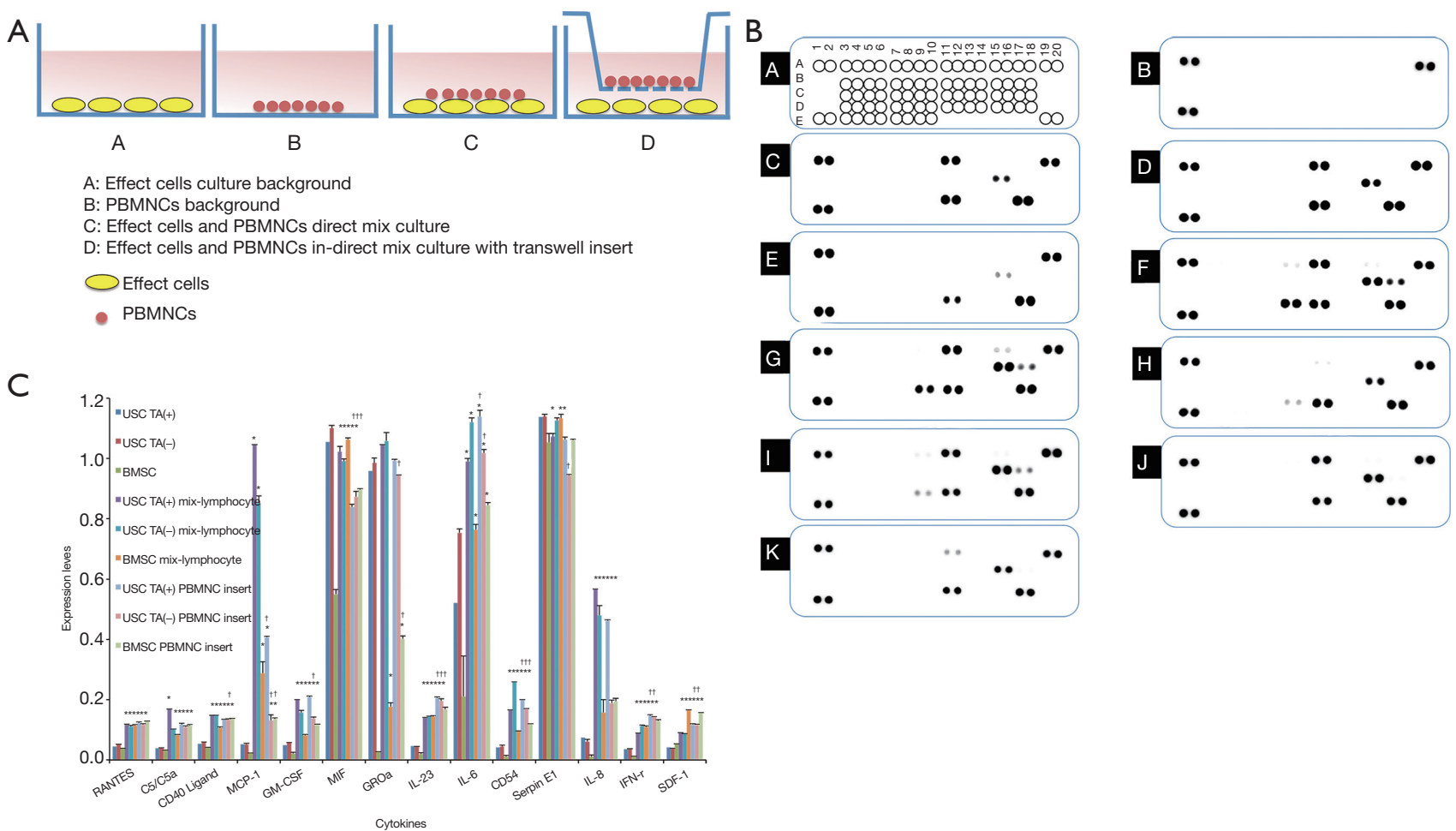

Figure 4 USC showed a characteristic cytokine release profile. The supernatants from USC-TA+, USC-TA-, and BMSCs cultured alone or co-cultured with PBMNCs (direct mixed culture with PBMNCs, or indirect mixed culture with PBMNCs in transwell insert for 48 h) (A) were assessed for their relative levels of cytokines and chemokines by using the human cytokine array panel A, as described in "Methods" (B). A: human cytokine array panel template; B: PBMNCs background; C: USC-TA+ culture alone; D: USC-TA- culture alone; E: BMSCs culture alone; F: USC-TA+ direct mix culture with PBMNCs; G: USC-TA- direct mix culture with PBMNCs; H: BMSCs direct mix culture with PBMNCs; I: USC-TA+ in-direct mix culture with PBMNCs in transwell insert; J: USC-TA- in-direct mix culture with PBMNCs in transwell insert; K: BMSCs in-direct mix culture with PBMNCs in transwell insert: The immunoblot of cytokine expression levels were visualized and quantifed $(\mathrm{C})$. $^{*}, \mathrm{P}<0.05$, comparing with BMSCs alone; $\uparrow, \mathrm{P}<0.05$, comparing direct and indirect mixed culture with PBMNCs. BMSC, human bone marrow mesenchymal stem cells; SMC, human smooth muscle cells; USC TA+, human urine derived stem cells with high telomerase activity; USC TA-, human urine derived stem cells with low telomerase activity; PBMNC, human peripheral blood mononuclear cells.

immunoregulatory cytokines and chemokines, especially IL-6, IL-8, and MCP-1, the correlation between elevated cytokine levels and USCs immunomodulatory properties and the underlying mechanism remain to be investigated. Furthermore, the immunomodulatory capacity of USCs needs to be evaluated in an in vivo model. All these concerns will be addressed in the following study.

\section{Conclusions}

This study we investigated the USCs immunomodulatory effects. Our results showed that USCs expressed typical MSC-like surface cell markers and possessed good immunomodulatory properties, comparable to those of BMSCs. Moreover, interacting with PBMNCs increased the secretion of immunoregulatory cytokines by USCs, especially with direct contacting with immune cells. Therefore, our findings provided a better understanding of the USCs immunomodulatory capacities for their potential application in tissue repair and cell therapy.

\section{Acknowledgments}

Funding: The study was supported by the Natural Science Foundation of Guangdong Province (grant number 2017A030313784). 


\section{Footnote}

Reporting Checklist: The authors have completed the MDAR reporting checklist. Available at https://dx.doi. org/10.21037/tau-21-506

Peer Review File: Available at https://dx.doi.org/10.21037/ tau-21-506

Conflicts of Interest: All authors have completed the ICMJE uniform disclosure form (available at https://dx.doi. org/10.21037/tau-21-506). The authors have no conflicts of interest to declare.

Ethical Statement: The authors are accountable for all aspects of the work in ensuring that questions related to the accuracy or integrity of any part of the work are appropriately investigated and resolved. This study was approved by the Wake Forest University institutional review board (IRB00014033). Written informed consents have been obtained and were approved by Wake Forest University institutional review board. The study was conducted in accordance with the Declaration of Helsinki (as revised in 2013).

Open Access Statement: This is an Open Access article distributed in accordance with the Creative Commons Attribution-NonCommercial-NoDerivs 4.0 International License (CC BY-NC-ND 4.0), which permits the noncommercial replication and distribution of the article with the strict proviso that no changes or edits are made and the original work is properly cited (including links to both the formal publication through the relevant DOI and the license). See: https://creativecommons.org/licenses/by-nc-nd/4.0/.

\section{References}

1. Munir H, McGettrick HM. Mesenchymal Stem Cell Therapy for Autoimmune Disease: Risks and Rewards. Stem Cells Dev 2015;24:2091-100.

2. Ray S, Sonthalia N, Kundu S, et al. Autoimmune disorders: an overview of molecular and cellular basis in today's perspective. J Clin Cell Immunol. 2013;S10. doi: 10.4172/2155-9899.S10-003.

3. Liang J, Wang D, Dominique F, et al. Mesenchymal stem cells for treating autoimmune diseases: The Chinese experience from lab to clinics. Curr Res Transl Med 2016;64:115-20.
4. Wei X, Yang X, Han ZP, et al. Mesenchymal stem cells: a new trend for cell therapy. Acta Pharmacol Sin 2013;34:747-54.

5. Wang L, Wang L, Cong X, et al. Human umbilical cord mesenchymal stem cell therapy for patients with active rheumatoid arthritis: safety and efficacy. Stem Cells Dev 2013;22:3192-202.

6. Bonab MM, Sahraian MA, Aghsaie A, et al. Autologous mesenchymal stem cell therapy in progressive multiple sclerosis: an open label study. Curr Stem Cell Res Ther 2012;7:407-14.

7. Wang D, Niu L, Feng X, et al. Long-term safety of umbilical cord mesenchymal stem cells transplantation for systemic lupus erythematosus: a 6-year follow-up study. Clin Exp Med 2017;17:333-40.

8. Zhou C, Wu XR, Liu HS, et al. Immunomodulatory Effect of Urine-derived Stem Cells on Inflammatory Bowel Diseases via Downregulating Th1/Th17 Immune Responses in a PGE2-dependent Manner. J Crohns Colitis 2020;14:654-68.

9. Yarygin KN, Lupatov AY, Sukhikh GT. Modulation of Immune Responses by Mesenchymal Stromal Cells. Bull Exp Biol Med 2016;161:561-5.

10. Friedenstein AJ, Piatetzky-Shapiro II, Petrakova KV. Osteogenesis in transplants of bone marrow cells. J Embryol Exp Morphol 1966;16:381-90.

11. Semon JA, Maness C, Zhang X, et al. Comparison of human adult stem cells from adipose tissue and bone marrow in the treatment of experimental autoimmune encephalomyelitis. Stem Cell Res Ther 2014;5:2.

12. Li JF, Zhang DJ, Geng T, et al. The potential of human umbilical cord-derived mesenchymal stem cells as a novel cellular therapy for multiple sclerosis. Cell Transplant 2014;23 Suppl 1:S113-22.

13. He X, Zhang Y, Zhu A, et al. Suppression of interleukin 17 contributes to the immunomodulatory effects of adiposederived stem cells in a murine model of systemic lupus erythematosus. Immunol Res 2016;64:1157-67.

14. Kolaparthy LK, Sanivarapu S, Moogla S, et al. Adipose Tissue - Adequate, Accessible Regenerative Material. Int J Stem Cells 2015;8:121-7.

15. Zhang $\mathrm{Y}, \mathrm{McNeill} \mathrm{E}$, Tian $\mathrm{H}$, et al. Urine derived cells are a potential source for urological tissue reconstruction. J Urol 2008;180:2226-33.

16. Bharadwaj S, Liu G, Shi Y, et al. Multipotential differentiation of human urine-derived stem cells: potential for therapeutic applications in urology. Stem Cells 2013;31:1840-56. 
17. Lang R, Liu G, Shi Y, et al. Self-renewal and differentiation capacity of urine-derived stem cells after urine preservation for 24 hours. PLoS One 2013;8:e53980.

18. Zhang D, Wei G, Li P, et al. Urine-derived stem cells: A novel and versatile progenitor source for cell-based therapy and regenerative medicine. Genes Dis 2014;1:8-17.

19. Schosserer M, Reynoso R, Wally V, et al. Urine is a novel source of autologous mesenchymal stem cells for patients with epidermolysis bullosa. BMC Res Notes 2015;8:767.

20. Kang HS, Choi SH, Kim BS, et al. Advanced Properties of Urine Derived Stem Cells Compared to Adipose Tissue Derived Stem Cells in Terms of Cell Proliferation, Immune Modulation and Multi Differentiation. J Korean Med Sci 2015;30:1764-76.

21. Vasu C, Wang A, Gorla SR, et al. CD80 and CD86 C domains play an important role in receptor binding and co-stimulatory properties. Int Immunol 2003;15:167-75.

22. Wen L, Zhu M, Madigan MC, et al. Immunomodulatory effects of bone marrow-derived mesenchymal stem cells on pro-inflammatory cytokine-stimulated human corneal epithelial cells. PLoS One 2014;9:e101841.

23. Solomon S, Pitossi F, Rao MS. Banking on iPSC-is it doable and is it worthwhile. Stem Cell Rev Rep 2015;11:1-10.

Cite this article as: $\mathrm{Wu} R$, Soland $M$, Liu G, Shi Y, Zhang C, Tang Y, Almeida-Porada G, Zhang Y. Functional characterization of the immunomodulatory properties of human urine-derived stem cells. Transl Androl Urol 2021;10(9):3566-3578. doi: 10.21037/tau-21-506
24. Nawa Y, Teshima T, Sunami K, et al. Responses of granulocyte colony-stimulating factor-mobilized peripheral blood mononuclear cells to alloantigen stimulation. Blood 1997;90:1716-8.

25. McIntosh K, Zvonic S, Garrett S, et al. The immunogenicity of human adipose-derived cells: temporal changes in vitro. Stem Cells 2006;24:1246-53.

26. Angoulvant D, Clerc A, Benchalal S, et al. Human mesenchymal stem cells suppress induction of cytotoxic response to alloantigens. Biorheology 2004;41:469-76.

27. Zhao Q, Ren H, Han Z. Mesenchymal stem cells: Immunomodulatory capability and clinical potential in immune diseases. J Cell Immunother 2016;2:3-20.

28. Rasmusson I, Ringdén O, Sundberg B, et al. Mesenchymal stem cells inhibit the formation of cytotoxic $T$ lymphocytes, but not activated cytotoxic $\mathrm{T}$ lymphocytes or natural killer cells. Transplantation 2003;76:1208-13.

29. Kyurkchiev D, Bochev I, Ivanova-Todorova E, et al. Secretion of immunoregulatory cytokines by mesenchymal stem cells. World J Stem Cells 2014;6:552-70.

30. Qin D, Long T, Deng J, et al. Urine-derived stem cells for potential use in bladder repair. Stem Cell Res Ther 2014;5:69. 